\title{
Direct micro-to-macro modelling of the cold rolling of pearlitic steel
}

\author{
Laurent Delannay $^{1, a}$, Jeroen Tacq ${ }^{2}$, Didier Bardel ${ }^{1}$ and Marc Seefeldt ${ }^{2}$ \\ ${ }^{1}$ iMMC, Université catholique de Louvain, 1348 Louvain la Neuve, Belgium \\ ${ }^{2}$ MTM, Katholieke Universiteit Leuven, 3001 Heverlee, Belgium
}

\begin{abstract}
A crystal plasticity model is adapted in order to predict strength, internal stresses and texture development in pearlite. The model is used as the user-defined material law in macroscopic finite element simulation of rolling. It is shown that the material response depends strongly on the reorientation of cementite lamellae. The model produces good estimates of the lattice strains measured by neutron diffraction as well as the uniaxial tensile curve.
\end{abstract}

\section{Introduction}

Metal forming processes such as rolling typically involve large plastic strains and significant changes of both the microstructure and the crystallographic texture. Such microstructural changes, which are a consequence of dislocation slip at the micron scale, may strongly influence the macroscopic plastic flow (e.g. the anisotropic hardening). In the present study, finite element (FE) modelling serves to predict strain heterogeneity across the thickness of a rolled workpiece, while the microscopic material response is computed based on an advanced crystal plasticity model.

The material under investigation is pearlitic steel [1], which has a characteristic dual-phase microstructure consisting of a thin stacking of ferrite and cementite (Fig. 1). Cementite forms a-few-nanometers-thick, extremely strong lamellae, whereas the strength of the softer ferrite depends on the inter-lamellar spacing. The composite response results from the contribution of both phases and it is expected to be anisotropic due to crystallographic texture and to the preferential alignment of cementite lamellae in the course of rolling.

The present study demonstrates that it is feasible to conduct direct micro-to-macro modelling of the microstructure (and strength) evolution during multi-pass rolling of pearlite. It is shown that texture and internal stresses are strongly influenced by the model assumptions about microscopic strain heterogeneity between the two constitutive phases as well as between adjacent grains (or adjacent pearlite "colonies").

\section{Model description}

Cold rolling simulations were performed following the procedure proposed by Bardel et al. [2] in order to study through-thickness texture gradients in rolled aluminium.

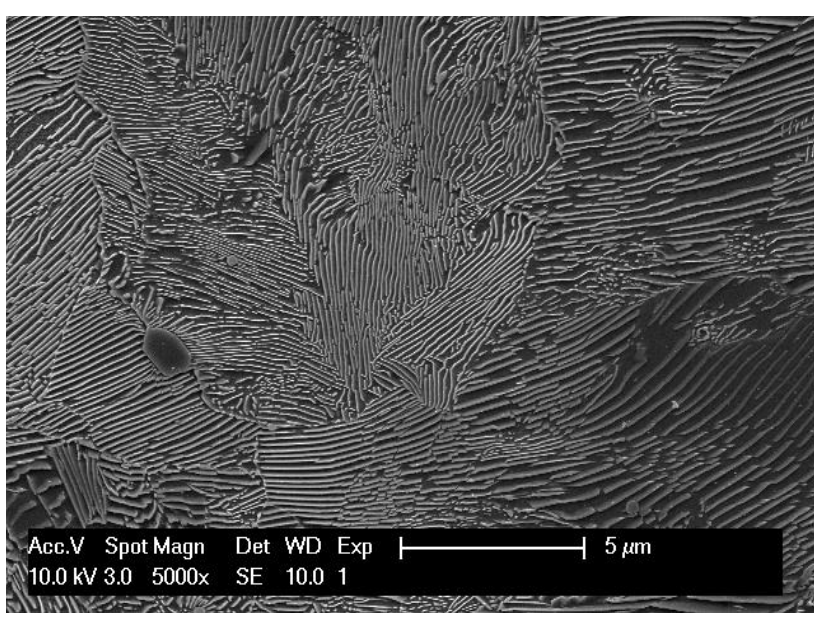

Figure 1. Microstructure of "patented" pearlite samples (before rolling). The cementite lamellae appear lighter. From [1].

Simulations, using the standard (implicit version of abaqus), assumed plane strain deformation, rigid rolls and finite relative displacement along the contact line between roll and workpiece. The half-thickness was discretized into 11 linear elements with reduced integration (C2D4R). Each rolling pass was simulated separately of the other passes, starting from square elements and using the state variables at the exit of a previous pass as input for the current pass (Fig. 2).

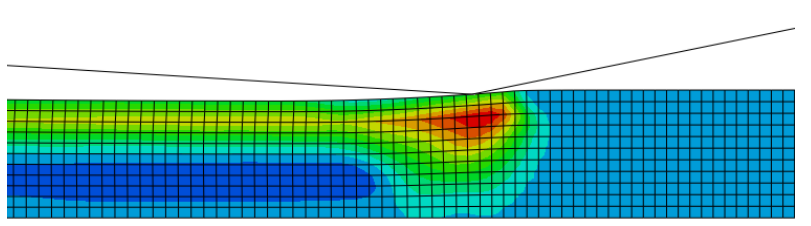

Figure 2. Illustration of the heterogeneity of shear strains across the workpiece thickness when steady state is detected at the exit of the rolling gap.

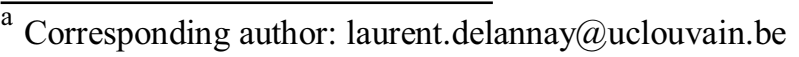


Inside one layer of elements across the thickness of the workpiece, the material response was computed using crystal plasticity theory. The model polycrystal consisted of 2000 grains and the interaction of adjacent grains was computed using the ALAMEL approximation [3-4]. This means that regions on either sides of a planar grain boundary underwent opposite shear superimposed to the macroscopic strain. The amount of the local strain heterogeneity was computed by minimizing the plastic work $[2,4]$. Within each grain crystal plasticity was applied to a stacking of ferrite and cementite lamellae (Fig. 3) while enforcing geometrical compatibility and stress equilibrium across the planar interface.

The initial lattice orientations were random in ferrite. The cementite lamellae were introduced by randomly selecting a variant of the Isaichev [1] orientation relationship within each grain. Hence, the interface normal was (110) in cementite and (112) in ferrite, whereas (010) in cementite was parallel to (111) in ferrite.

Dislocation glide occurred along $\{110\}<111>$ and $\{112\}<111>$ systems in ferrite, whereas $(010)[001]$ and (110)[111] type slip systems were considered in cementite [5] which has orthorhombic symmetry $(a=$ $5.0816 \AA, b=6.7446 \AA, c=4.5206 \AA)$. An isotropic Voce representation of hardening was used in each phase:

$$
\tau_{c}=\tau_{s a t}-\left(\tau_{s a t}-\tau_{c 0}\right) \exp \left(\Gamma / \Gamma_{0}\right)
$$

where $\tau_{c 0}$ and $\tau_{s a t}$ were the initial and the saturating value of the critical resolved shear stress (CRSS) $\tau_{c}$, whereas $\Gamma$ was the accumulated slip on all slip systems and $\Gamma_{0}$ determined the initial hardening rate.

Time integration of the crystal plasticity law was fully implicit and a consistent tangent operator was provided to the FE solver $[2,4]$.

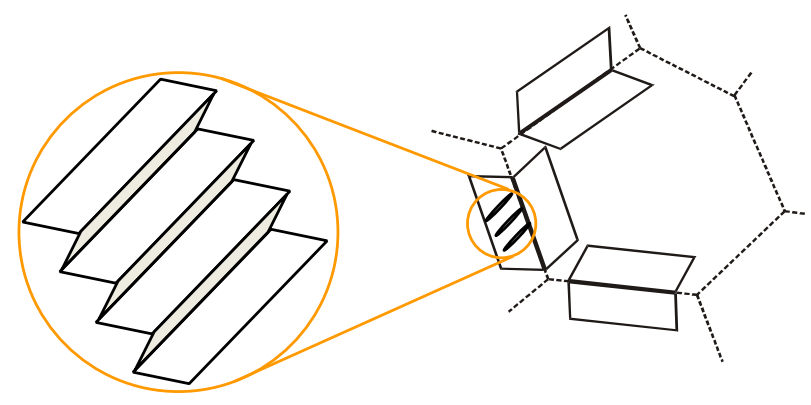

Figure 3. Representation of the strain heterogeneity considered between adjacent grains (right) and within each grain due to the stacking of soft ferrite and hard cementite (left).

\section{Results}

The experimental data used to assess the model was obtained based on uniaxial tensile tests on dog-boneshaped samples of C75 fully pearlitic steel sheet with a cementite volume fraction of about $12 \%$. Tests were done after patenting at $500^{\circ} \mathrm{C}$, giving a mean inter-lamellar spacing of $140 \mathrm{~nm}$, and after cold rolling to an equivalent strain of 1.1 with subsequent stress-relief treatment at $470^{\circ} \mathrm{C}$ for $15 \mathrm{~min}$. The tensile axis was aligned with the rolling direction [2].

\subsection{Determination of the hardening parameters}

The elastic constants and the hardening parameters of the two phases are listed in Table 1. The elastic constants of (embedded) cementite have been assumed as equal to the ones of ferrite; the hardening parameters have been determined by fitting the experimental tensile curves obtained on the pearlite samples before and after rolling (Fig. 4). The same set of parameters was thus used for the simulation of the two tensile tests as well as the intermediary rolling.

Table 1. Material parameters.

\begin{tabular}{|c|c|c|}
\hline & Ferrite & Cementite \\
\hline$C_{11}, C_{12}, C_{44}[\mathrm{GPa}]$ & $231,135,116$ & $231,135,116$ \\
\hline$\tau_{\mathrm{c} 0}[\mathrm{MPa}]$ & 250 & 1600 \\
\hline$\tau_{\mathrm{sat}}[\mathrm{MPa}]$ & 380 & 2000 \\
\hline$\Gamma_{\square}[-]$ & 65 & 10 \\
\hline
\end{tabular}

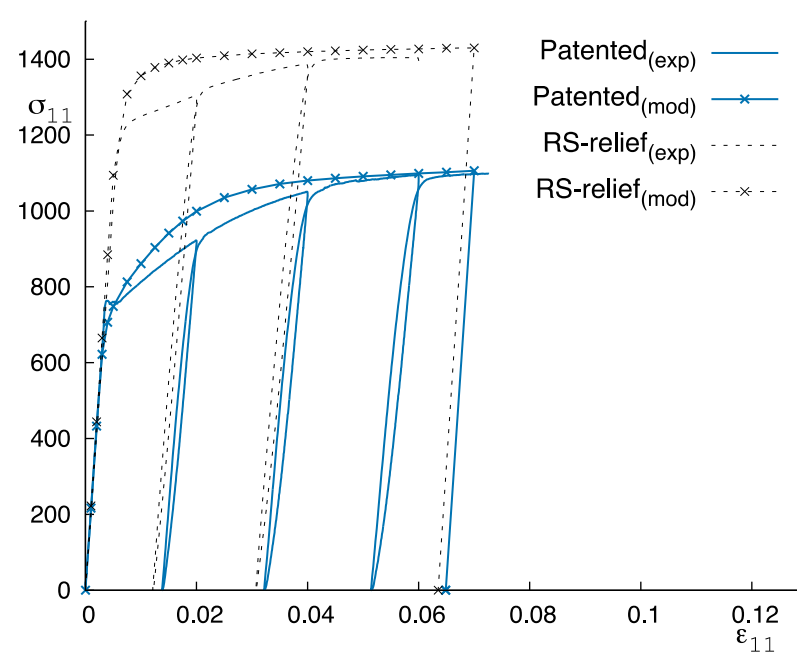

Figure 4. Uniaxial tensile stresses [MPa] obtained on pearlite samples before ("patented") and after cold rolling to a strain of 1.1 (and partial relaxation of internal stresses "RS-relief").

The initial CRSS value for ferrite is in the range that one would expect for an as-patented material and the present mean inter-lamellar spacing: Gil Sevillano [6] proposed, based on the assumptions of intralamellar dislocation glide in the ferrite channels and predominant activation of intralamellar multiplicative dislocation sources, a lower and an upper bound of the critical shear stress of

$$
\tau_{b} \cong 1.2 \frac{G b}{2 \pi S} \ln \frac{s}{b}
$$

and

$$
\tau_{m} \cong 1.2 \frac{G b}{\pi S} \ln \frac{s}{2 b}
$$


respectively. For the present mean interlamellar spacing $S$ this gives a critical shear stress range from about 175 to about $315 \mathrm{MPa}$. Note that these estimates do neither include Peierls stress, nor solution strengthening, nor dislocation storage strengthening, compare, for instance, [7]. With appropriate update of the interlamellar spacing, however, they can form the basis of a physics- and microstructure-based hardening law [8]. The initial and saturation values for cementite correspond to estimated cementite strengths of about 5-8 GPa reported in literature $[6,9]$.

\subsection{Internal stresses}

Internal stresses developing during the two uniaxial tensile tests have been measured in situ using neutron diffraction (in transmission) on the strain diffractometer beamline HK9 at the Nuclear Physics Institute of ASCR, Řež near Prague, Czech Republic. Tensile rig and sample were positioned such that the tension and rolling direction was aligned with the diffraction vector (Young setup) and the entire width of the sample was immersed in the neutron beam. In the plastic regime, each neutron diffraction measurement was done at constant extensometer strain, implying some stress relaxation during the measurement. The ferrite lattice strains in tensile and rolling direction were monitored for the $<200>,<211>,<220>$ and $<310>$ orientation fibres. Three unloading-reloading cycles were carried out in order to study possible reverse yielding of ferrite [1].

Fig. 5 demonstrates that the crystal plasticity model produced fair predictions of the longitudinal lattice strains in different crystallographic fibres. The model predictions presented in these plots correspond to the average elastic strain developed in the tensile direction in the sampling of grains oriented in such a way that a chosen crystal direction is tilted by less than $7^{\circ}$ from the tensile axis.

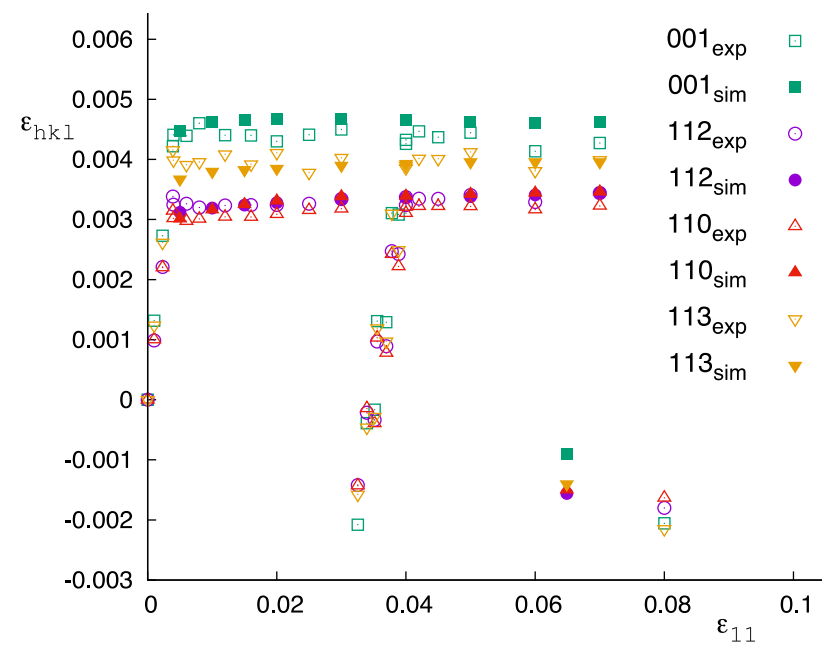

(a)

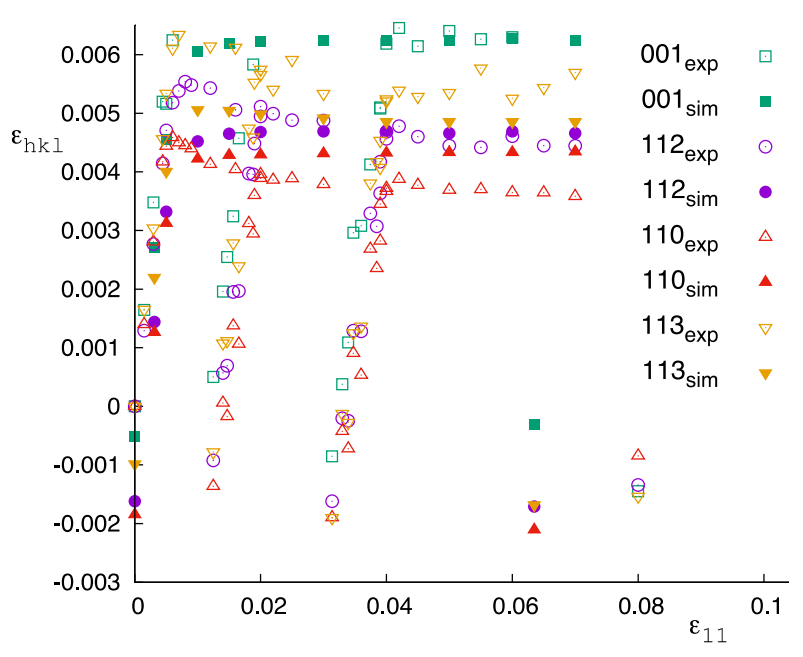

(b)

Figure 5. Assessment of the model predictions of the elastic lattice strains during the uniaxial tensile test on (a) the patented sample and (b) the cold rolled sample (after a heating treatment relaxing the internal stresses due to rolling).

\subsection{Cold rolling texture}

The measured and predicted textures of ferrite after cold rolling are presented in Fig. 6. The predicted texture development was somewhat too rapid, leading to larger intensities of the Orientation Distribution Function (ODF) as compare to experiment. Yet the model predicted the correct stable texture components.
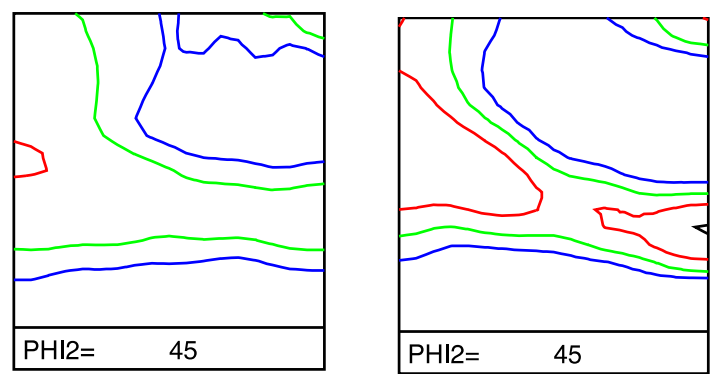

Figure 6. Section $\varphi_{2}=45^{\circ}$ of the orientation distribution function (ODF) of the ferrite phase after cold rolling. The experimental texture is on the left and the predicted one is on the right. The contour lines intensities are: $1-2-4-8$ [10].

\section{Discussion}

In Fig. 4, the macroscopic tensile curves are reproduced only approximately when using a Voce law to represent the strain hardening of dislocation slip systems. The model overestimates the yield stress but it predicts a valid estimate of the strength after a small elongation. The larger overestimation of the yield stress of the cold rolled and stress-relieved sample may be expected because the stress relief treatment will have resulted in some recovery due to dislocation annihilation and rearrangement, which is not incorporated in the Voce law as it was used for both cold rolling and subsequent tensile straining. Moreover, both the cold rolled and the stress-relieved samples show an extended micro yield regime, which 
may be due to microstructural heterogeneity, as revealed by SEM. The fact that the deviation is limited and fading out with increasing strain thus indicates that the recoverable part of work-hardening linked to dislocation storage remains small compared to the non-recoverable one linked to lamellae narrowing and alignment.

The ferrite lattice strains measured during the tensile tests by in situ neutron diffraction are globally well predicted (Fig. 5). In particular, the ordering of the reflections is properly reproduced within the range of experimental uncertainty for the loading part of both curves. This ordering can be understood as a consequence of elastic anisotropy: the $<200>$ grain family is the most compliant one and thus develops the highest lattice strains; the $<110>$ grain family is the stiffest one and thus develops the lowest lattice strains. With increasing plastic deformation one would expect gradual load transfer from the softer $<200>$ and $<310>$ orientation fibres towards the stronger $<110>$ and $<211>$ orientation fibres. However, within the limited plastic strain range of the test, this is observed neither in the experiment, nor in the simulations. This might be an indication that for plastic anisotropy of ferrite the morphology of cementite is the dominating factor over the lattice orientation of ferrite.

The model does not cover the observed slight relaxations after yielding of the cold rolled and stressrelieved sample. In this sample, the ferrite and cementite lamellae are largely aligned with the rolling plane. The ferrite cold rolling texture is almost fully developed. Ferrite softening might then be due to (micro) shear banding and/or due to rupture of some of the cementite lamellae, both not incorporated in the model. The model does capture the average amplitude of the lattice strains that reflect the residual stress state after unloading (bottom-right of the plots), but here the ordering of the diffraction peaks is incorrect.

The cold rolling texture of ferrite (at the sheet midthickness) is correctly simulated but its intensity is larger than the intensity of the experimental texture (Fig. 6). It is possible that such discrepancy is due to the localization of plastic deformation in microscopic shear bands (MSB). The influence of MSB on the evolution of microstructure and texture of pearlite is currently under investigation, using MSB model suggested in [11].

An important feature of the model considered here is that it accounts for the progressive reorientation of the cementite lamellae during rolling. Whereas cementite lamellae are randomly oriented in the initial model polycrystal, they then tend to align parallel to the rolling plane (Fig. 7). As shown in Fig. 8, this contributes significantly to the macroscopic strength of the rolled sheet. Indeed, cementite lamellae are loaded less when they are randomly oriented than when they are aligned parallel to the tensile direction.

The model predictions rely of the simplified ALAMEL assumption about the interaction of adjacent grains. Crystal plasticity based finite element modelling (CPFEM) is likely to provide a more accurate reproduction of the grain-to-grain strain heterogeneity [4]. This has been checked by modeling the pearlite polycrystal with a finite element mesh in which each grain was represented by cubic element with eight integration points. Fig. 9 compares the phase stress obtained using either the ALAMEL model or CPFEM, demonstrating that the difference is rather small.

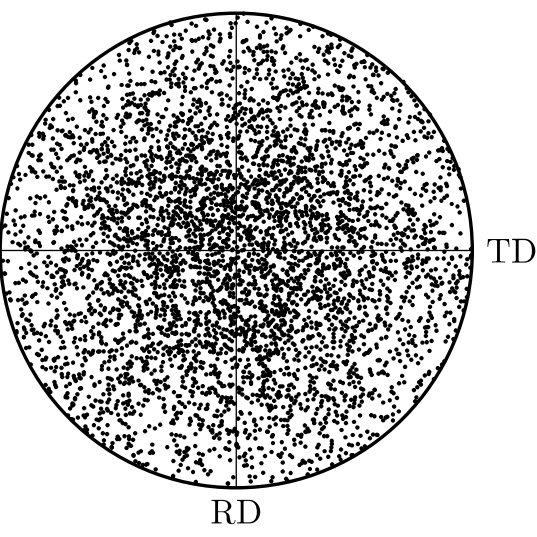

(a)

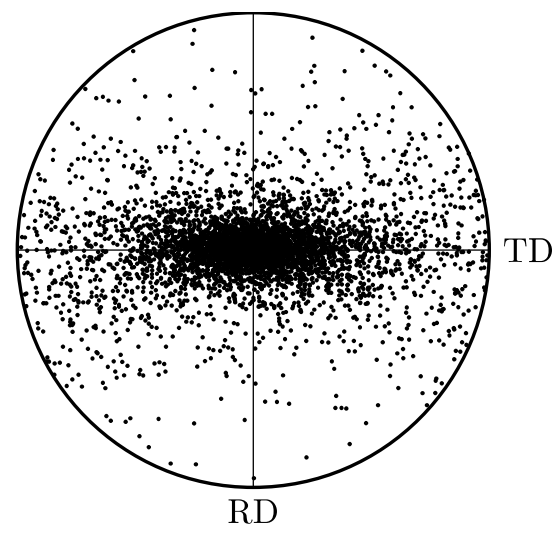

(b)

Figure 7. Pole figures showing the distribution of orientations of the cementite lamellae relative to the rolling direction (RD), the transverse direction (TD) and the sheet normal direction (ND) before (a) and after (b) rolling.

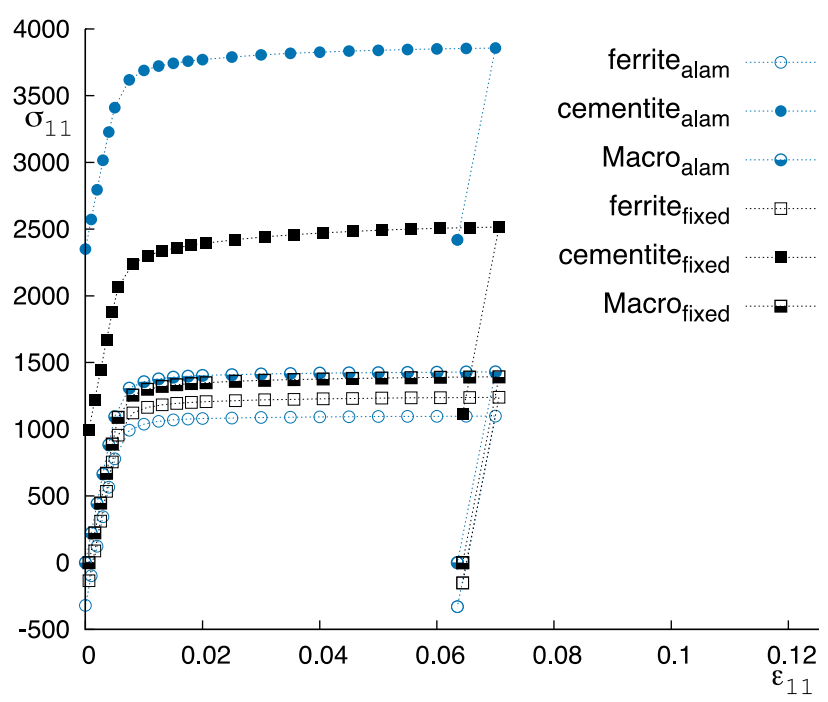

Figure 8. Model prediction of the phase and macroscopic stresses $[\mathrm{MPa}]$ when cementite lamellae are either re-oriented during rolling or not (resp. "alam" or "fixed"). 


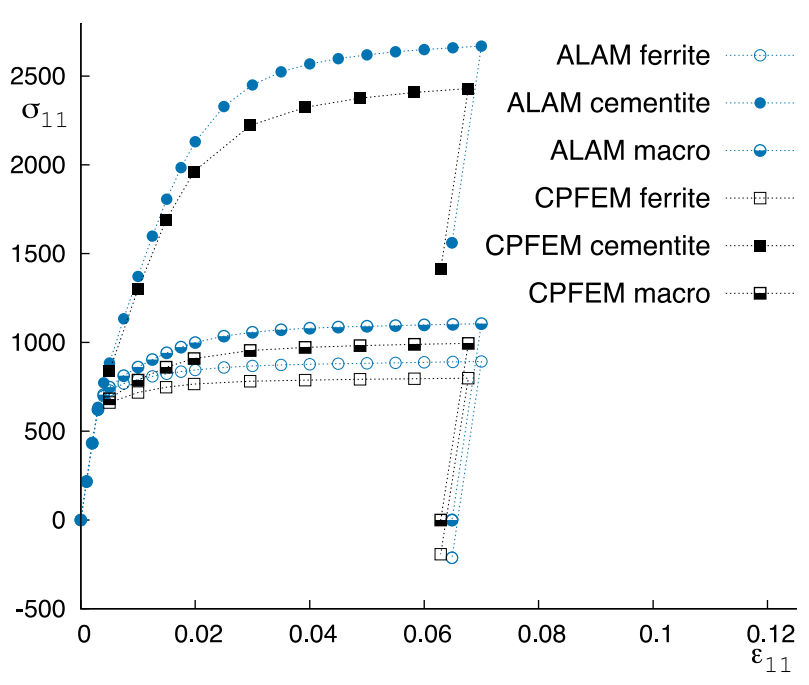

Figure 9. Model prediction of the phase and macroscopic stresses [MPa] when using either the ALAMEL model or CPFEM.

The microscopic CPFEM prediction can be improved by using more than a single finite element to represent each grain. However, an explicit representation of pearlite lamellae would imply refining the mesh so much that the computation time would become excessive.

\section{Conclusion}

A crystal plasticity model has been adapted in order to account for the cooperative deformation of ferrite and cementite phases in pearlite. The model has been implemented as a user-defined material law in a finite element code allowing macro scale simulation of rolling. Comparison of the predictions to experimental measurements of internal stresses and texture indicate that the mechanical response is strongly influenced by the progressive rotation of cementite lamellae, which preferentially align parallel to the rolling plane. Accounting for such microstructural evolution during metal forming is a strong motivation for the development of micro-to-macro modelling schemes.

\section{Acknowledgements}

Funding was obtained through the IAP project 7/21 "Multiscale Mechanics of Interface Dominated Materials" of the Belgian Science Policy BELSPO. LD is mandated by the FSR-FNRS, JT held a fellowship of The Research Foundation - Flanders (FWO). The neutron diffraction experiments were conducted at the Czech Center of Accelerators and Nuclear Analytical Methods (CANAM, LM2011019) with funding from the Ministry of Education, Youth and Sports of the Czech Republic. CANAM is gratefully acknowledged for granting beam time and support, in particular through the beam time scientist Dr. Jan Pilch. The European Union's Seventh Framework Program for research, technological development and demonstration supported the neutron experiments under the NMI3-II Grant number 283883.

\section{References}

1. J. Tacq, Residual and Internal Stress Development Resulting from Plastic Deformation of Multi-Phase Alloys: the Case of Pearlite. Ph.D. Thesis, KU Leuven, Belgium (2015).

2. D. Bardel, L. Delannay, O.V. Mishin, Int. J. Plast. (in preparation).

3. P. Van Houtte, S. Li, M. Seefeldt, L. Delannay, Int. J. Plast., 21 (2005), 589-624.

4. L. Delannay, M.A. Melchior, J.W. Signorelli, J-F. Remacle, T. Kuwabara, Comp. Mater. Sci., 45 (2009), 739-743.

5. J. Gil Sevillano, J. Mater. Sci. Eng., 21 (1975), 221.

6. J. Gil Sevillano, J. Phys. III, 1 (1991), 967-988.

7. X. Zhang, A. Godfrey, X. Huang, N. Hansen, Q.Liu, Acta mater., 59 (2011), 3422-3430.

8. P. Watté, P. Van Houtte, E. Aernoudt, J. Gil Sevillano, W. Van Raemdonck, I. Lefever, Mater. Sci. Forum, 157-162 (1994), 1689-1694.

9. Y. Tomota, P. Lukáš, D. Neov, S. Harjo, Y.R. Abe, Acta mater., 51 (2003), 805-817.

10. P. Van Houtte, MTM-FHM software, Department MTM, KU Leuven, Belgium (2015).

11. F. Lin, L. Delannay, J. Mech. Phys. Sol. (submitted). 